\title{
Development Concepts for Mars Ascent Vehicle (MAV) Solid and Hybrid Vehicle Systems
}

\author{
Lisa Tunstill McCollum \\ NASA Marshall Space Flight \\ Center \\ Mail Code: ER52 \\ Huntsville, AL 35812 \\ 256-544-5052 \\ lisa.k.mccollum@nasa.gov
}

\author{
Andrew Schnell \\ NASA Marshall Space Flight \\ Center \\ Mail Code: ED04 \\ Huntsville, AL 35812 \\ 256-544-8913 \\ andrew.schnell@nasa.gov
}

\author{
Rachel McCauley \\ NASA Marshall Space Flight \\ Center \\ Mail Code: ED04 \\ Huntsville, AL 35812 \\ 256-544-8332 \\ rachel.j.mccauley@nasa.gov
}

\author{
Darius Yaghoubi \\ NASA Marshall Space Flight \\ Center \\ Mail Code: EV41 \\ Huntsville, AL 35812 \\ 256-544-7718 \\ darius.f.yaghoubi@nasa.gov
}

\begin{abstract}
The Advanced Concepts Office (ACO) at Marshall Space Flight Center (MSFC) has conducted ongoing studies and trades into options for both hybrid and solid vehicle systems for potential Mars Ascent Vehicle (MAV) concepts for the Jet Propulsion Laboratory (JPL).
\end{abstract}

Two MAV propulsion options are being studied for use in a potential Mars Sample Retrieval (MSR) campaign. The following paper describes the current concepts for hybrid and solid propulsion vehicles for MAV as part of a potential MSR campaign, and provides an overview of the ongoing studies and trades for both hybrid and solid vehicle system concepts. Concepts and options under consideration for vehicle subsystems include reaction control system (RCS), separation, and structures will be described in terms of technology readiness level (TRL), benefit to the vehicle design, and associated risk.

A hybrid propulsion system, which uses a solid fuel core and liquid oxidizer, is currently being developed by JPL with support from MSFC. This type of hybrid propulsion vehicle would allow the MAV to be more flexible at the cost of higher complexity, in contrast to the solid propulsion vehicle that is simpler, but allows less flexibility.

The solid propulsion vehicle study performed by MSFC in 2018 further refined the solid propulsion system sizing as well as added definition to vehicle subsystem concepts, including the RCS, structures and configuration, interstage and separation, aerodynamics, and power/avionics.

The studies were performed using an iterative concept design methodology, engaging subject matter experts from across MSFC's propulsion and vehicle systems disciplines as well as seeking trajectory feedback from analysts at JPL.
TABLE OF CONTENTS

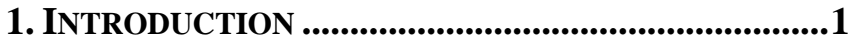

2. CONCEPT DESIGN METHODOLOGY .........................3

3. HYBRID MAV DESIGN OVERVIEW ..........................5

4. SOLID MAV DESIGN OVERVIEW ...........................6

5. CONCEPT COMPARISON, CONCLUSIONS, AND

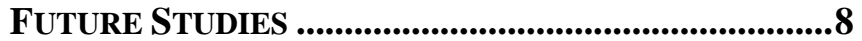

ACKNOWLEDGEMENTS ..........................................9

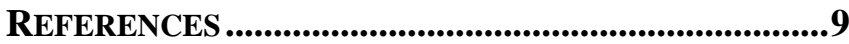

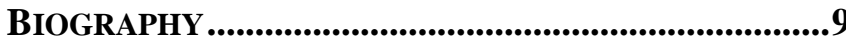

\section{INTRODUCTION}

As NASA's Mars Ascent Vehicle (MAV) project prepares to choose a propulsion system for use on the MAV for a potential Mars Sample Retrieval (MSR) campaign, Marshall Space Flight Center's (MSFC) Advanced Concepts Office (ACO) has been tasked with performing concept and trade studies for hybrid propulsion and solid propulsion MAV systems.

After two years of focusing on increasing maturity and characterization of the hybrid propulsion system, the MAV project team desires to reassess, in terms of risk, the options of propulsive launch vehicles for use in a MAV. The concepts created will be used to enable comparison and be downselected by the MAV and MSR projects to a single propulsion system to move forward to a detailed design. This paper describes the scope and relevance of the study, the design methodology and developed concepts, the details of the solid and hybrid propulsion system concepts, and the general comparisons and conclusions of the study. 


\section{MSR Campaign Architecture}

A potential robotic MSR campaign is being studied by the Jet Propulsion Laboratory (JPL) in Pasadena, California. This prospective MSR campaign would consist of several elements (Figure 1), including:

- A sample caching rover to collect geological samples from the surface of Mars

- A sample retrieval lander to bring a Mars Ascent Vehicle to transport the samples off the surface of Mars

- An Earth return orbiter to receive the samples for transport back to Earth

Current estimates put dwell time on the surface of Mars at approximately six months, where the MAV would arrive on the surface of Mars and be stored on the lander platform until geological samples were secured onboard.
Previous Studies-MSFC has been involved in multiple MAV feasibility studies since 2011, focusing on three propulsion systems: solid rocket motors, liquid rocket engines, and hybrid rocket motors. ${ }^{1}$ In 2016, the MSR team shifted focus and resources to the technological development of hybrid propulsion - the least mature of the three optionsto advance the technology readiness level (TRL) and gain a better understanding of system performance and mass.

\section{Reassessing MSR Propulsion Systems for Risk Reduction}

In 2018, JPL requested that MSFC reassess the risk associated with the three propulsion technologies, liquid, solid, and hybrid. The eight-week study began in March 2018 , led by MSFC's ACO in partnership with subject matter experts from various engineering organizations across the center. Part of MSFC's JPL-delegated task was to update the concepts for comparable solid and liquid propulsion MAVs using identical assumptions to the current hybrid MAV

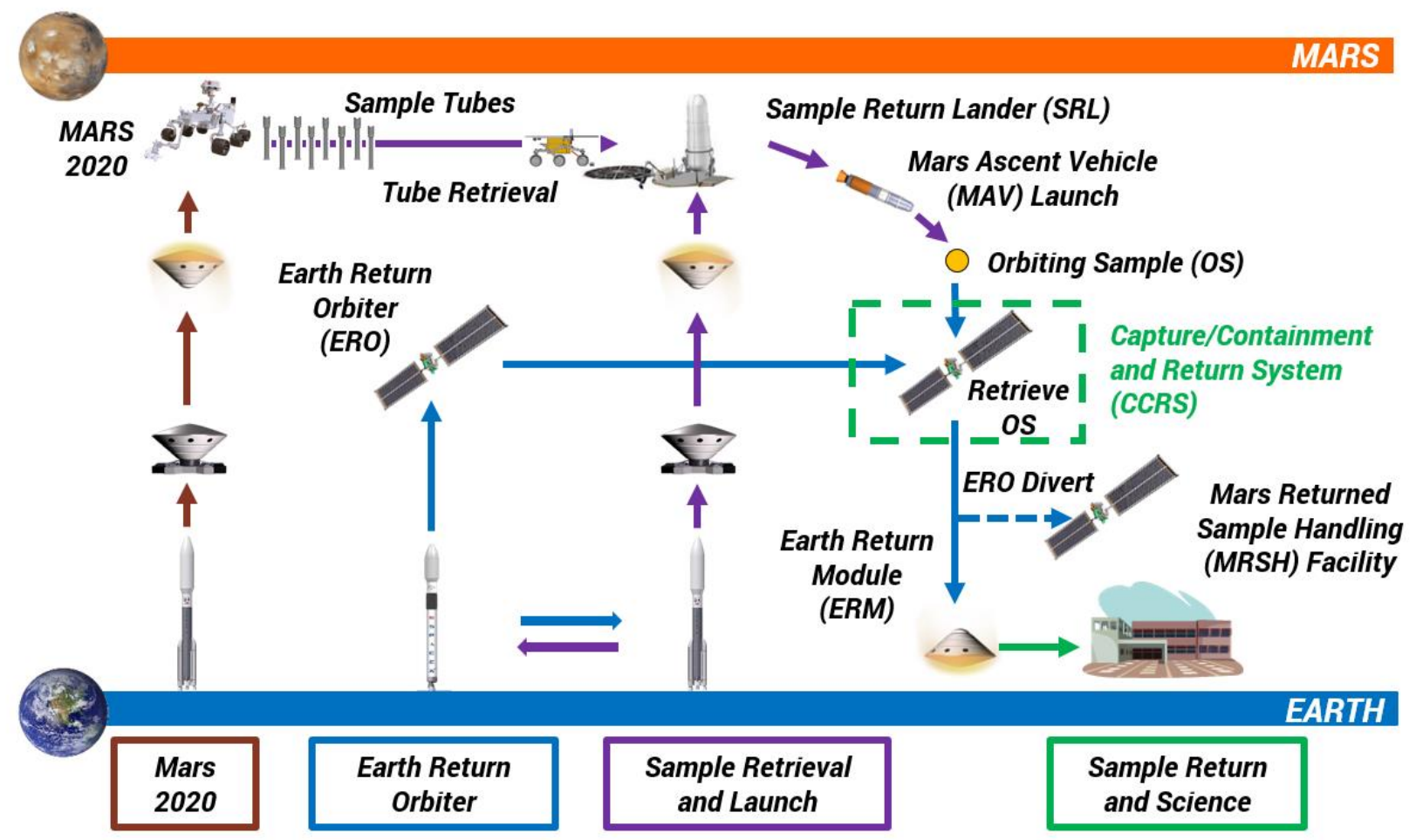

Figure 1. Mars Sample Retrieval Concept of Operations

Challenges-The MSR campaign architecture presents multiple design challenges to a small launch vehicle, including the cold storage environment on the surface of Mars, packaging limitations of the lander, and the delivery performance and trajectory requirements for transporting samples to Mars orbit. In light of these challenges, several options for risk mitigation and technology maturation were identified and developed over the past several years of feasibility studies.

\footnotetext{
${ }^{1} 1$-stage and 2-stage vehicle configurations have been considered for all three propulsion types.
}

concept wherever possible to enable an accurate comparison between the technologies for MAV application.

ACO cooperated closely with other organizations across MSFC's engineering directorate for conceptual aspects that required special definition or maturity. For the duration of the concept study, JPL provided the trajectory analysis and evaluation of each concept design iteration. Although the entire team was not co-located, the short timeframe for the study did not allow for any significant dwell time between 
design and analysis cycles. Virtual work software enabled meetings with real-time iteration between MAV vehicle design updates at MSFC and trajectory assessments at JPL.

The ACO's resulting solid and liquid concepts were presented at the MAV Peer Review in May 2018. The liquid MAV concept had issues meeting the volume and mass constraints, so the ACO continued development on the solid MAV concept as a risk reduction strategy for the MSR program.

About the Diverse Concept Study Team-The MSFC MAV concept study was led by the ACO in close cooperation with various engineering organizations within MSFC, their MAV formulation office, and the JPL MAV team. MSFC's ACO has a rich history performing the up-front conceptual designs for many successful space missions, performing complete and integrated systems analyses and independent assessments of potential concepts, as well as technology assessments and subsystem trades for a variety of projects. [1]

The ACO staffs multiple subject matter experts with close ties to their respective discipline areas, and this expertise was instrumental to the concepts for main propulsion and reaction control systems (RCS) sizing, avionics system assessment, power system sizing, and configuration design. MSFC is home to many technical specialties for launch vehicle design and analysis, such as solid rocket motor grain design, RCS thruster selection, interstage separation, vehicle structures, guidance, navigation, and control (GNC) and launch vehicle stability management.

\section{CONCEPT DESIGN Methodology}

\section{Design Methodology and Primary Constraints}

The concept study for the solid propulsion MAV was performed using ACO's iterative design methodology. The study began by examining the hybrid propulsion MAV ground rules, assumptions, and constraints to identify a similar set for a solid MAV concept in order to facilitate an effective comparison between the two vehicle designs.

The primary constraints were the mass of the payload, orbit to be achieved, and the physical envelope on the lander that the MAV would be limited to (mass and volume). After creating a rough vehicle sizing for mass and performance, various subsystems analyses were quickly run to determine impacts. The vehicle sizing was updated based on the subsystems feedback. This cycle was repeated many times with emphasis on various subsystems and interfaces to approach a realistic and more optimized vehicle concept.

It is important to note that all vehicle subsystems were not optimized during this short concept study. Analysis was prioritized by those subsystems which were most uncertain or presented the most risk to mission safety or vehicle mass. Some subsystems remained approximations at the completion of the concept study, with documented recommendations for forward work to mature them.

\section{Performance Needs and Architecture Assumptions}

For the solid MAV concept, performance needs drove the team to a 2-stage propulsion vehicle configuration. This in turn necessitated additional subsystems such as a structural interstage and stage separation system.

The primary constraints for the concept design study revolved around performance needs to deliver the sample payload to the specified orbit, along with the mass and volume limitations for stowage of the MAV on the lander. Other variations such as the non-operational temperature and Mars atmosphere entry acceleration loads were considered, but would require more analysis than was performed during the initial 8-week study. A list of ground rules, assumptions and constraints developed for the concept study by the MSFC study team in cooperation with JPL is found in Table 1.

\section{Table 1. Ground Rules, Assumptions and Constraints} for MAV Concept Study

\begin{tabular}{|c|c|}
\hline Parameter & Assumption/Constraint \\
\hline \hline Orbital Insertion Height & $343 \mathrm{~km}$ \\
Orbital Insertion Inclination & $18-25^{\circ}$ \\
Payload Mass & $18 \mathrm{~kg}$ \\
Vehicle Mass & $400 \mathrm{~kg}$ (max.) \\
Vehicle Length & $3 \mathrm{~m}$ (max.) \\
Vehicle Diameter & $0.57 \mathrm{~m}(\max )$. \\
Non-Operational Temp. & $-70{ }^{\circ} \mathrm{C}$ to $+40{ }^{\circ} \mathrm{C}$ \\
Operational Temp. & $-20{ }^{\circ} \mathrm{C}$ \\
Entry Acceleration Loads & $15 \mathrm{~g}$ (lateral) \\
Angle of Attack & $0.2^{\circ}$ to $3.3^{\circ}$ \\
Post-Insert Divert Maneuver & No divert maneuver \\
RCS Location & Fwd of $2^{\text {nd }}$ stage / within OML \\
Avionics/Power Hardware & Maximum similarity to hybrid \\
Performance Margin & Addtl. 5 kg payload \\
Other Margin Allocation & AIAA margins / 25\% for \\
& unknowns \\
\hline
\end{tabular}

\section{Avionics and Power Sizing}

As both hybrid and solid MAV concepts are further studied and matured, the avionics and power systems will be traded with additional options outside the components considered in this concept design study. Avionics and power subsystems for a solid motor design were assessed by ACO's subject matter experts for similarities to the hybrid vehicle design. The study team recommended alterations to make the hybrid design's avionics and power hardware work for the solid MAV concept design - for example, the interstage separation system on the solid MAV requiring additional controller hardware compared to the hybrid, single-stage MAV. This approach was used for the best possible comparison to the hybrid MAV by the Peer Review described in Section 1. 


\section{Structures Sizing Estimation}

The basic structure size estimate for the solid MAV concept design was derived from configuration design geometry. The iterative nature of the study and frequently changing vehicle configuration allowed a generalized structural analysis and optimization for the vehicle structures.

Material properties and thicknesses were chosen based on previous, similarly-sized launch vehicles studied by MSFC's engineering directorate. Applying these material properties and notional thicknesses to the configuration geometry allowed for an estimation of structures mass. 25\% margins were used in most vehicle area structures due to the uncertainty inherent to the estimation methodology.

\section{Main Propulsion System (MPS) Sizing}

For the solid MAV concept, performance needs and the results of multiple past studies quickly led the team to a twostage vehicle configuration. ${ }^{2}$ The first- and second-stage solid rocket motors were sized according to this estimate and the resulting performance and mass was reported back for incorporation into JPL's trajectory analysis.

MPS sizing iteration was performed multiple times as vehicle masses and performance changed, as detailed in [2]. A concern about vehicle controllability during the maximum dynamic pressure event arose during an iteration of propulsion sizing and trajectory analysis. If maximum dynamic pressure occurred at or near first-stage burn out, the vehicle lost the controllability afforded by the MPS thrust vector control (TVC) during a critical time.

Working closely with MSFC's solid rocket motor design experts, the team was able to quickly design a realistic thrust profile for the first stage motor that provides for TVC controllability at maximum dynamic pressure and does not burn out until the dynamic pressure was much lower. [2]

\section{Reaction Control System (RCS) Sizing}

Sizing of the RCS system was performed based on estimated aerodynamic loads. It was assumed that the first- and secondstage TVC will maintain vehicle trajectory during MPS burns; the RCS will only be used for adjustments during the 500 -second coast period between MPS burns. In order to achieve the smallest and lightest RCS system possible, the outer mold line (OML) of the MAV was adjusted in order to attain the most aerodynamically stable vehicle possible. This minimized the amount of perturbations that the RCS needed to work to correct, and resulted in a concept with a much smaller RCS system. [2]

\footnotetext{
${ }^{2}$ Propulsion system sizing began with a $\Delta \mathrm{V}$ and $\Delta \mathrm{V}$-split estimate from JPL's trajectory analysis.

${ }^{3}$ The structural study began with current estimates for hybrid and
}

\section{Ongoing Updates as Technology Matures}

It is expected that assumptions and constraints will continue to shift as technology maturation continues across the MSR architecture. Aspects were changed during the course of the MAV concept study, and will continue to change as various elements of the MSR architecture are studied further. Significant changes that occurred late in the study were noted in the final concept presentation and documented for further examination in the next phase of study.

For some subsystems, multiple options are identified and documented for purposes of either risk mitigation or the ability to easily perform sensitivity assessments in the next phase of studies. Particularly, the team was able to identify more than one feasible option for the interstage separation system and the RCS system. The team documented additional options for further consideration as the MAV concepts become more mature and program requirements and constraints continue to evolve.

As an interim step to increase the maturity of the vehicle structures designs for both the hybrid and solid MAV concepts, detailed structural analysis was performed during a study in the summer of $2018 .^{3}$

The structural masses quoted in this paper do not reflect the recent structural analysis. The structural estimates from summer 2018 will be picked up by the 2019 study team and finalized, at which time new concepts for both the hybrid and solid MAV will be reported.

\section{Additional Considerations and Interfaces of Concern}

While the MAV concept itself presents a challenging design to close, the MAV within the larger context of the potential MSR campaign presents additional considerations and challenges. The interfaces with other pieces of the MSR architecture are elements that the MAV design must consider and react to when there are changes. Primary interfaces of concern are:

- The lander on which MAV will be stowed until launching from the surface of Mars

- The Orbiting Sample (OS), which will need to be accessed and/or installed during the dwell time on Mars

- The Mass Payload Adapter (MPA), the structural element that houses the OS

- The Earth Return Orbiter (ERO) that will receive the OS and transport it back to Earth

These interfaces are at various levels of maturity. For all, but particularly the less mature interfaces, the MAV study team

solid MAV structures and replicated the geometry in finite element models, using HyperSizer, a structural sizing optimization tool, to optimize the structures for minimal mass. 


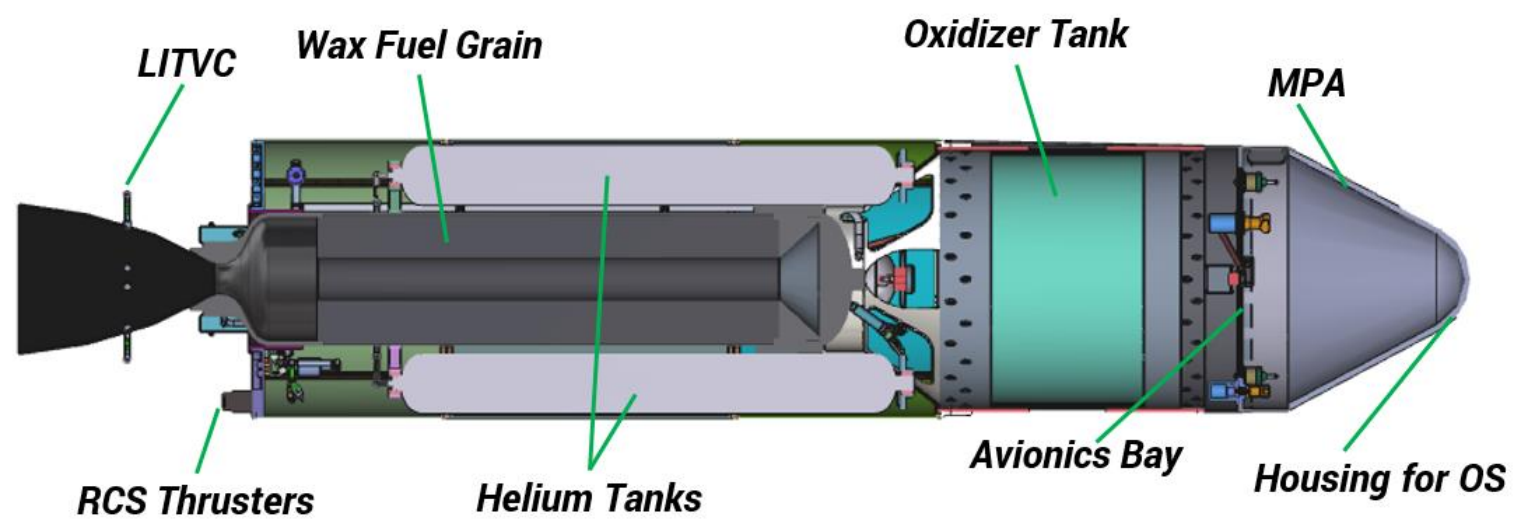

Figure 2. Hybrid MAV Concept Design

has the opportunity to provide input, which may make the architecture more successful and reduce risk.

\section{HYBRID MAV DESIGN OVERVIEW}

Figure 2 illustrates the configuration of the hybrid propulsion MAV design. The hybrid MAV is a single stage to orbit (SSTO) vehicle that uses a solid fuel grain made of SP7 wax and a liquid oxidizer that contains a high percentage of mixed oxide of nitrogen (MON). The MON oxidizer is also used for a liquid injection TVC (LITVC), which injects the MON into the exhaust plume of the hybrid motor to adjust the direction of thrust and maintain vehicle trajectory. A cold gas RCS system with thrusters mounted on the aft of the motor uses Helium gas, already on board for use as pressurant for the MON, to create thrust for both vehicle roll control and for finer adjustments needed during the approximate 500 -second coast period.

\section{Mass}

Table 2 summarizes the total Gross Lift-Off Mass (GLOM) and subsystem masses for the hybrid MAV. Some of the subsystems, including avionics and structure, are derived from a previous test program and therefore represent some uncertainty with regard to mass required for the MSR architecture. These vehicle subsystems require further analysis and maturation to reduce the mass risk to the MAV.

Table 2. Mass Summary for Hybrid MAV Concept

\begin{tabular}{|c|c|}
\hline Element & Mass $(\mathbf{k g})$ \\
\hline \hline Hybrid MAV GLOM & $\mathbf{3 7 2}$ \\
Reserve Mass & 5 \\
Payload & 18 \\
Avionics \& Telecom & 4 \\
Power & 0.4 \\
Vehicle Structure \& & 12 \\
Thermal & 296 \\
Propellant & 36 \\
Propulsion Dry Mass & \\
\hline
\end{tabular}

\section{Performance}

The performance parameters for the hybrid MAV concept are summarized in Table 3.

Table 3. Performance Summary for Hybrid MAV

\begin{tabular}{|c|c|}
\hline Parameter & \\
\hline \hline$\Delta \mathrm{V}(\mathrm{m} / \mathrm{s})$ & appx. 4000 \\
Total Impulse (Ns) & 824,300 \\
Specific Impulse (s) & 308 \\
Average Thrust (N) & 6830 \\
\hline
\end{tabular}

\section{RCS Concept}

The current RCS concept for the hybrid MAV leverages the Helium gas already onboard for use as the MON oxidizer pressurant. The two pods of tri-directional thrusters use cold Helium gas blow-down to generate thrust for RCS. Cold gas RCS is a high-TRL option with many examples of successful use in space.

In the 2019 studies outlined in Section 6, the RCS needs for the hybrid vehicle will be further evaluated in order to ensure optimal sizing for performance and minimal excess mass. A better understanding of the aerodynamic stability of the hybrid MAV vehicle is critical in consideration of the RCS performance needs. This analysis has not yet been completed and will be studied in detail in the 2019 studies.

\section{TVC}

The hybrid MAV concept uses a LITVC system to maintain vehicle trajectory. The LITVC system relies on additional MON oxidizer being injected into the exhaust plume of the motor in order to modify thrust. The LITVC system has been successfully tested in preliminary development tests of the hybrid propulsion system using low-percentage MON.

Currently, the LITVC concept has a lower TRL than other more commonly used TVC systems. In order to mature the concept, the LITVC system will continue to be tested in static 


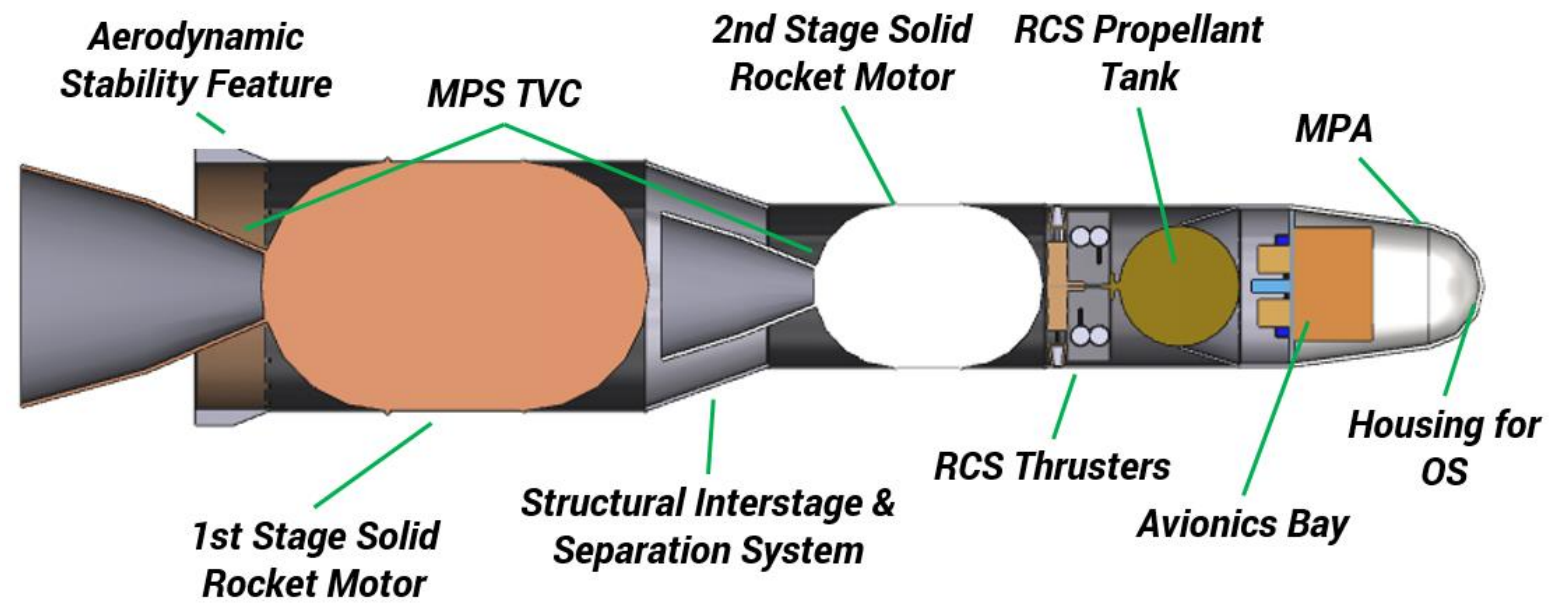

Figure 3. Solid MAV Concept Design

development tests of the hybrid propulsion system, and the data from these tests will be analyzed by TVC experts. Any adjustments to the LITVC concept due to this testing and analysis will be incorporated into the 2019 studies described in Section 6.

\section{Vehicle Structures Considerations}

Structural analysis, to date, relies on several assumptions including constraints and detailed characteristics of features on the lander interface. The JPL team charged with designing the lander provided a geometric model of the current concept for the interface, which was replicated in a finite element model. The primary load cases for structural design are the $15 \mathrm{G}$ lateral load imparted by parachute deployment during lander descent at Mars, and the $9 \mathrm{G}$ load in any direction during landing on the Mars surface. The lander supports must be sufficient to carry these loads and support the MAV during these events.

Additionally, the launch guide structure is a critical component to be considered in relation to the MAV vehicle structure.. Not only must the mass be minimized, the launch guide structure must be sufficient to guide the MAV during ignition and initial phase of launch. The current concept for launch guidance involves two rails on a series of structural rings. The MAV would reside inside the structural rings and upon launch initiation, be guided by the rails away from the lander.

\section{Hybrid MAV Challenges}

To date, the development testing for the hybrid propulsion system has used low-percentage MON. Because the concept design for the flight vehicle uses high-percentage MON, it is important to perform further testing of the hybrid propulsion system using high-percentage MON in order to reduce risk and properly characterize performance.

Additionally, aerodynamic stability and RCS control authority are important areas to be addressed for the hybrid
MAV. Because the TVC does not provide trajectory control during the approximately 500-second coast period between MPS burns, the RCS must be robust enough to maintain trajectory during this time.

\section{SOLID MAV DESIGN OVERVIEW}

Figure 3 illustrates the configuration of the solid propulsion MAV design. The solid MAV is a two stage vehicle that uses solid rocket motors derived from heritage designs used for inspace missions. Each solid rocket motor would contain its own electro-mechanical TVC system and controller. A hydrazine monopropellant RCS with thrusters packaged above the second stage motor would provide the finer adjustments needed during the estimated 500-second coast period.

\section{Mass}

Table 4 summarizes the total Gross Lift-Off Mass (GLOM) and subsystem masses for the hybrid MAV. Some of the subsystems, including avionics and structure, are derived from a previous test program and therefore represent some uncertainty with regard to mass required for the MSR architecture. These vehicle subsystems require further analysis and maturation to reduce the mass risk to the MAV.

\section{Table 4. Mass Summary for Solid MAV Concept}

\begin{tabular}{|c|c|}
\hline Element & Mass (kg) \\
\hline \hline Solid MAV GLOM & $\mathbf{3 7 4}$ \\
Reserve Mass & 5 \\
Payload & 18 \\
Avionics \& Telecom & 10 \\
Power & 0.5 \\
Vehicle Structure \& Thermal & 31 \\
Propellant & 263 \\
Propulsion Dry Mass & 47 \\
\hline
\end{tabular}




\section{Performance}

The performance parameters for the solid MAV concept are summarized in Table 5.

Table 5. Performance Summary for Solid MAV Concept

\begin{tabular}{|c|c|}
\hline Parameter & $\mathbf{1}^{\text {st }}$ Stage / $\mathbf{2}^{\text {nd }}$ Stage \\
\hline \hline$\Delta \mathrm{V}(\mathrm{m} / \mathrm{s})$ & appx. 4000 \\
Total Impulse (Ns) & $620,730 / 113,230$ \\
Specific Impulse (s) & 291 \\
Average Thrust (N) & $13,794 / 4,355$ \\
\hline
\end{tabular}

\section{RCS Concept}

The current RCS concept for the solid MAV is a monopropellant hydrazine system. The selection of a hydrazine system was driven by aerodynamic stability calculations. By estimating aerodynamic perturbations expected during the approximately 500 -second coast period between first- and second-stage burns and assuming a duty cycle, it was possible to estimate the magnitude of thrust and total propellant needed from RCS to maintain trajectory. This calculation is further detailed in [2].

There is a desire to further evaluate whether there can be a return to a cold gas (Helium or similar) RCS system as it would possibly be smaller and pose less of a handling risk. Further evaluation of the aerodynamic stability of the vehicle is required in order to further optimize this system.

\section{TVC}

The solid MAV concept uses an electromechanical actuatordriven TVC system based on TVC systems used extensively on tactical solid rocket motors. The primary advantage of this system is its high TRL due to extensive operational use and resulting well-understood parameters and high reliability. This TVC system was studied extensively in the 2011-2015 studies on solid propulsion concepts for MAV and that research was leveraged to benefit the 2018 concept study. This system could be purchased "off-the-shelf" with minimal modifications needed for the MAV application.

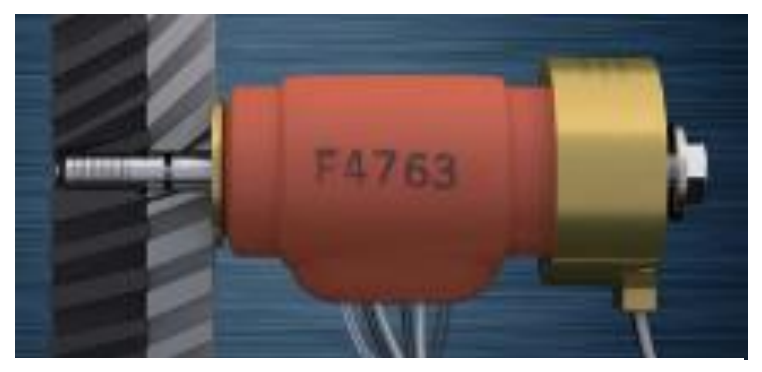

Figure 4. FC4 Frangibolt Actuator

\section{Interstage Separation Concept}

The primary option for the interstage separation mechanism is a high-TRL "frangibolt" actuator system. This system is a commercially available mechanism, and is non-pyro and lowshock. The frangibolt actuator is illustrated in Figure 4. The advantages to this system are primarily its relatively high TRL and the safety afforded by the non-pyro nature of the mechanism. There is little risk to the nozzle due to fragmentation and low risk to the payload samples due to pyro shock. The disadvantage of this system is that it is relatively heavy and accounts for over 4 kilograms of the estimated structural mass.

Other options for interstage separation that were considered in the concept study and should continue to be evaluated as the concepts mature include:

- A typical robust linear shape charge

- A commercially available spring separation system

- A conceptual ultra-lightweight isotruss clamshell with spring separation

Each additional option brings with it considerations for TRL, mass, and safety risk to the rest of the vehicle. Additionally, further consideration of the placement of the separation plane is required to minimize shock to the payload, nozzle clearance, and stage mass requirements.

\section{Vehicle Structures Considerations}

Many structural considerations for the solid MAV are the same or similar to the considerations and constraints for the hybrid MAV. The primary differences for the solid MAV lie in the challenges presented by the unique shape of the current concept for the OML. The significant change in diameter at the interstage presents a challenge for structural support and launch guidance within the current concept for the lander interface.

\section{Solid MAV Challenges}

The potential for extreme, cold temperatures in the Mars storage environment presents an unknown for solid rocket motors. Propellant grain, insulation, and the structural integrity of other soft goods at extreme, low temperatures have not been characterized to the level needed for this mission. Additional work to characterize the structural behavior of the solid rocket motors is necessary.

The OML of the current solid vehicle presents a challenge for lander support and interfaces, although it improves aerodynamic stability. Further study needs to be done on the structural supports, launch guides, and interfaces with the lander in order to arrive at a vehicle OML and lander interface that sufficiently provides structural support and guides the MAV during launch while retaining acceptable mass properties and vehicle aerodynamic stability. 


\section{CONCEPT COMPARISON, CONCLUSIONS, AND FUTURE STUDIES}

\section{Solid and Hybrid Concept Comparison}

A summary comparison of hybrid and solid MAV concepts is presented in Table 6 .

Table 6. Comparison of Key Parameters of Solid and Hybrid MAV Concepts

\begin{tabular}{|c|c|c|}
\hline Parameter & Hybrid MAV & $\begin{array}{c}\text { Solid MAV } \\
\mathbf{1}^{\text {st }} \text { Stage } / \mathbf{2}^{\text {nd }} \text { Stage }\end{array}$ \\
\hline \hline GLOM (kg) & $\mathbf{3 7 4}$ & $\mathbf{3 7 4}$ \\
Total Impulse (Ns) & 824,300 & $620,730 / 113,230$ \\
Sp. Impulse (s) & 308 & 291 \\
Average Thrust (N) & 6830 & $13,794 / 4,355$ \\
Avionics \& & 4 & 10 \\
Telecom (kg) & & 0.5 \\
Power (kg) & 0.4 & 31 \\
Vehicle Structure \& & 12 & 263 \\
Thermal (kg) & & 47 \\
Propellant (kg) & 296 & \\
Propulsion Dry & 36 & \\
Mass (kg) & & \\
\hline
\end{tabular}

GLOM-As shown in Table 6, The hybrid and solid are of similar GLOM. The specific impulses are somewhat similar, although the hybrid is more efficient. While the numbers reported here show a significantly higher mass for the solid vehicle structures than for the hybrid vehicle structures, it is noted that structural design is currently in work and will continue through the 2019 studies to develop more realistic estimates of structural mass for both vehicle concepts.

$T R L$ - In terms of TRL, the hybrid motor remains a mid-TRL technology. There have been significant gains in the maturity of the hybrid propulsion system over the last two years of development activities by the MAV project, but still requires significant work to characterize its performance and risk in the context of an MSR campaign. Solid rocket motors are a high-TRL option that have been extensively qualified to cold temperatures (although not to the extremes expected for an MSR campaign) in tactical systems and used successfully in space missions.

Bridging the gap in characterization between the operational environments experience in historical applications and the expected MSR environments remains the primary work of maturing the solid propulsion system for the MAV. This makes the solid propulsion MAV concept an appropriate risk reduction option for consideration by the MAV project in 2019.
Temperature Performance in Expected EnvironmentsAnother important consideration for comparison of the MAV concepts includes temperature performance in the expected Mars environment. As noted in Section 4, further characterization of the performance of the solid rocket motors in such cold temperatures is needed. The hybrid propulsion system will potentially store and perform better in the extreme thermal environment expected.

\section{Interfaces and Mission Architecture Considerations}

For either MAV concept, the interfaces with the lander present a challenge that will continue to be studied and negotiated for the remainder of concept development. Lander mass constraints mean that the physical envelope for MAV stowage requires the MAV to minimize excess mass as much as possible while still meeting performance. Similarly, lander power constraints mean that limited heating can be provided to the MAV while stowed on the surface of Mars. The MAV must make design decisions considering the extreme temperature environments on the surface of Mars as described in Table 1.

Finally, structural support and launch guides are a challenge for the lander design. The MAV team must continually stay abreast of any changes to the lander design, constraints, and assumptions in order to ensure that the MAV design still closes with any change to the interfaces.

\section{Future Studies}

The decision regarding propulsion system to use for the MAV will be made in late 2019. To facilitate this decision, MSFC's Advanced Concepts Office is leading two design studies in parallel. One design study will consider a MAV with a conventional solid rocket motor, and the second will consider a MAV with the proposed hybrid solid rocket motor with oxidizer.

The two design studies will be similar in scope to previous studies. Subject matter experts will break down the MAV requirements and size each system to meet them. The two propulsion systems will be designed using the data from models, historical test data, and data from upcoming hot-fire testing to be completed in early 2019. The results of the studies will be a pair of MAV concepts, one for each propulsion system. Each design will have power, mass, and cost estimates. The interfaces with the lander will be defined. Impacts to risk posture, schedule, and budget will be estimated. This information will aid the decision-makers in deciding which propulsion system to proceed with.

As time permits, the design team will consider how each propulsion concept could meet the requirements of a lunar ascent vehicle for a lunar sample retrieval mission. A lunar sample retrieval mission using a similar propulsion system would be an opportunity to reduce the risk for the Mars sample retrieval mission. 


\section{ACKNOWLEDGEMENTS}

The authors wish to thank the MSFC MAV project team for their continued support, Mike Baysinger and Sharon Fincher from MSFC's Advanced Concepts Office for providing images of MAV concepts, the MSR and MAV design teams at JPL for their continued support, Erin Lanigan from MSFC's Materials and Processes Laboratory for her structural analysis, George Story and Britt Oglesby from MSFC's Propulsion Systems Department for their support of the hybrid system design, and Krista Haraway and Rebekah Frederick from MSFC's Advanced Concepts Office for their technical writing support.

\section{REFERENCES}

[1] Marshall Space Flight Center's Advanced Concepts Office Website:

https://www.nasa.gov/centers/marshall/capabilities/advan ced_concepts.html

[2] Bean, Q. et al., "Mars Ascent Vehicle (MAV) Propulsion Subsystems Design." IEEE Aerospace Conference, Big Sky, MT, 2018 (abstract submitted)

\section{BIOGRAPHY}

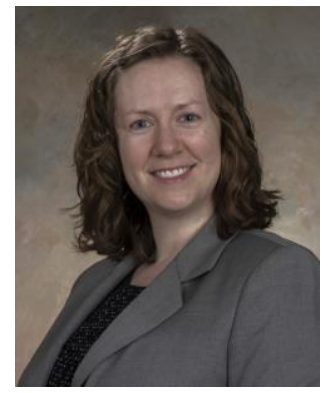

Lisa Tunstill McCollum received her Bachelor's Degree in Mechanical and Aerospace Engineering in 2010 from the University of Alabama in Huntsville and soon after began her career in systems engineering supporting trade studies for the evolution of the Ballistic Missile Defense System as a contractor with the Missile Defense Agency. Mrs. McCollum has worked for NASA since 2012 in the solid propulsion systems division where she has served as project engineer and then re-design lead for an in-house designed sounding rocket sustainer motor, and currently serves as NASA's Abort Motor lead for Orion's Launch Abort System. During a recent detail to Marshall Space Flight Center's Advanced Concepts Office, she acted as study lead for Mars Ascent Vehicle (MAV) concept studies. In addition to her NASA duties, Mrs. McCollum is active in the North Alabama section of the American Society of Mechanical Engineers (ASME) and values volunteering her time encouraging young people to pursue engineering careers.

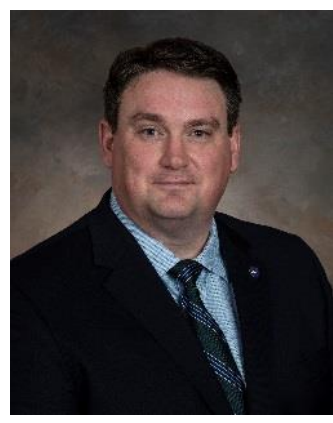

Andrew Schnell is a study lead and thermal system designer for Marshall Space Flight Center's Advanced Concepts Office. In over six years in Advanced Concepts, he has led ACO's design efforts on a variety of conceptual design studies including the Lynx X-Ray Observatory, Mars sample return missions, the Europa lander de-orbit stage, the HabEX telescope, and SLS's Exploration Upper Stage. He has also contributed thermal designs for deep space habitats, interstellar probes, solar sails, satellites, cubesats, SLS payloads, and ISS experiments. Prior to joining Advanced Concepts, Andrew was a member of NASA's Cryogenic Fluid Management team, where he managed the design and preparation of several cryogenic test articles. He holds a patent for the design of novel foam-rigidized inflatable structures. Andrew is a graduate of Tennessee Technological University.

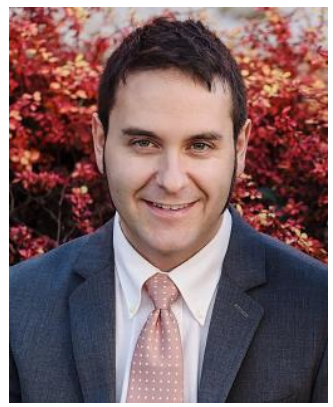

Darius Yaghoubi began working for NASA in June 2007, shortly after graduating from North Carolina State University with a Bachelor's of Science in Aerospace Engineering. Upon starting work in the Control Systems Design \& Analysis Branch (EV41), Darius spent most of his time working on 
launch vehicle design. In addition to providing support for launch vehicle separation and liftoff analysis, Darius also completed external work details in loads and dynamics, avionics/software, propulsion systems testing, systems engineering for Deep Space Habitat, and structural/mechanical design. In 2011, Darius was appointed as the lead pogo stability analyst on SLS, a position that he still holds today. In 2015, Darius was placed in the position of Branch Team Lead, providing him a leadership position to act as a liaison between engineers and management. In 2018, Darius began work as the Guidance, Navigation, and Control lead for the Mars Ascent Vehicle (MAV) and has since transitioned to the role of Vehicle Technical Lead. Aside from his technical work, Darius is an active member of the MSFC Speaker's Bureau and has represented NASA at a number of public outreach and speaking events such as the 7th Wernher von Braun Memorial Symposium, NASA on the Square, and countless career days at local and nation-wide schools.

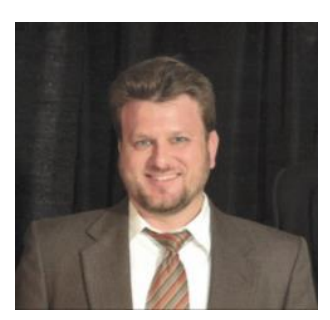

Quincy Bean graduated from the University of Southern California with a Master of Science in Astronautical Engineering in 2013. He has worked at NASA for 5 years and began his career as a Materials Engineer specializing in Additive Manufacturing. While there he served as the Principal Investigator and Technology Development Lead for the In-Space Manufacturing Project. He currently serves as the Propulsion Technology Lead in the Advanced Concepts Office where he designs propulsion systems for a variety of vehicle and mission concepts.

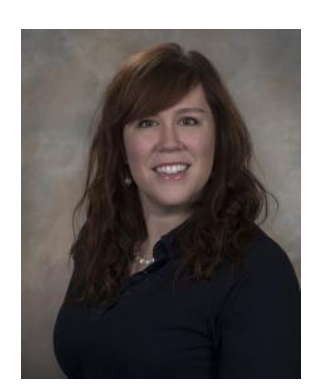

Rachel McCauley is the Deputy Manager of the Advanced Concepts Office at NASA's Marshall Space Flight Center in Huntsville, Alabama. She received her master's degree in Aerospace Engineering in 2012 and her undergraduate degree in Chemical Engineering in 2003, both at the University of Alabama in Huntsville. During her career at NASA she has been a propulsion engineer and led several rocket design teams, including the Orion Launch Abort System's three solid rocket motors and the Robotic Mars Ascent Vehicle. Rachel has also served as an independent engineering representative with experience evaluating risk to ensure the feasibility of technical and program project plans and designs.

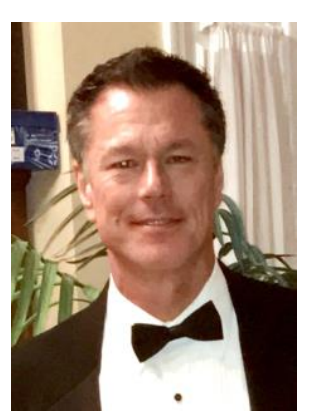

Andrew Prince has worked in the solids and hybrids industry for 30 years with OATK and NASA. He served as the Nozzle Chief Engineer for the Ares $1^{\text {st }}$ stage and was the NASA technical lead for the Orion Launch Abort System. Recently he has taken part in the design and manufacture of the Peregrine Sounding Rocket and concept design of the Mars Ascent Vehicle first stage. Mr. Prince has a patent for a method of composites manufacture and 12 published papers with JANNAF and AIAA on material test beds, and solid and hybrid propulsion systems. Currently he is working as solid propulsion lead for the MARS 2020 MAV vehicle development team. 